\title{
Drenagem torácica em condições traumáticas e não traumáticas e suas complicações
}

\section{Tube thoracostomy in traumatic and non-traumatic conditions and its complications}

\author{
1 Daniel Camargo de Jesus Araújo danicamargo2112@gmail.com \\ 1 Isabella Moura Silva \\ 1 Renato Camargo de Jesus Araújo \\ 2 Eliane Camargo De Jesus \\ 1 Centro Universitário de Volta Redonda, UniFOA.
2 Pós doutorado em oncologia pelo AC Camargo Câncer Center. Centro Universitário de Volta Redonda, UniFOA.
}

\section{Resumo}

A drenagem torácica pode ser um procedimento para salvar vidas, se a função cardiorrespiratória estiver comprometida. O objetivo deste trabalho foi descrever as principais indicações de toracostomia encontradas em condições traumáticas e não traumáticas e suas complicações. Trata-se de um estudo transversal, realizado no Hospital Santa Casa de Misericórdia de Barra Mansa. Foi utilizada uma amostra consecutiva, com pacientes submetidos à drenagem torácica, realizada no centro cirúrgico, no período de junho de 2016 a junho de 2018. As principais indicações de toracostomia encontradas neste estudo foram traumática $(37.0 \%)$ e neoplásica (22.2\%). A taxa de complicação pós-toracostomia foi de $22.2 \%$ neste estudo, sendo, a mais comum, pneumonia $(7.4 \%)$, seguida de empiema e pneumotórax $(5.6 \%$ cada). O sucesso do procedimento depende da técnica correta durante sua realização e dos devidos cuidados pós-operatórios. As possíveis complicações apresentam incidência aceitável e, na maioria das vezes, são facilmente tratadas.

Palavras-chave:

Complicações. Fatores de risco. Toracostomia.

\begin{abstract}
Tube Thoracostomy can be a life-saving procedure if cardiorespiratory function is compromised. The aim of this study was to describe the main indications for thoracostomy found in traumatic and non-traumatic conditions and its complications. This is a cross-sectional study carried out at Hospital Santa Casa de Misericórdia de Barra Mansa. A consecutive sample was used with patients undergoing tube thoracostomy performed in the operating room from June 2016 to June 2018. The main indications for thoracostomy found in this study were traumatic (37.0\%) and neoplastic (22.2\%). The rate of post-thoracostomy complication was $22.2 \%$ in this study, the most common was pneumonia (7.4\%) followed by empyema and pneumothorax (5.6\% each). The success of the procedure depends on the correct technique during its performance and the appropriate post-operative care. The possible complications have an acceptable incidence and in most cases are easily treated.
\end{abstract}

Keywords:

Complications. Risk factors. Thoracostomy.

Como você deve citar?

ARAÚJO, Daniel Camargo de Jesus et al. Drenagem torácica em condições traumáticas e não traumáticas e suas complicações. Cadernos UniFOA, Volta Redonda (RJ), v. 16, n. 47, p. 139 - 145, dez, 2021. 
A drenagem torácica é um procedimento de grande importância em pacientes que apresentam traumas ou outras lesões dentro da cavidade pleural, principalmente se a função cardiorrespiratória estiver comprometida. A abordagem comumente aceita recomenda o acesso ao espaço pleural através do triângulo de segurança da British Thoracic Society (BTS) em uma área delimitada pela dobra axilar, superiormente; o nível do mamilo ou sulco inframamário, inferiormente; grande dorsal, posteriormente; e borda lateral do peitoral maior, anteriormente (PATRICK et al., 2018).

A toracostomia com tubo (TT) é atualmente um tratamento amplamente utilizado para pneumotórax, hemotórax, hemopneumotórax, empiema pleural e derrames pleurais, sendo um dos procedimentos terapêuticos invasivos mais realizados (VILKKI e GUNN, 2019). Apesar da drenagem pleural ser considerada como ato relativamente simples, pode levar a severas complicações, devido à falta de cuidados que precisam ser respeitados (CIPRIANO, 2011).

As complicações da TT podem ser classificadas como técnicas ou infecciosas. As causas técnicas incluem o mal posicionamento do tubo, o bloqueio do dreno, o dobramento do dreno do tórax, o edema pulmonar de reexpansão, o enfisema subcutâneo, lesões nervosas, lesões cardíacas e vasculares, lesões esofágicas, pneumotórax residual / pós-intubação, fístulas, recorrência tumoral no local de inserção, hérnia através do local, quilotórax e disritmias cardíacas. As complicações infecciosas incluem empiema e infecção no local cirúrgico, incluindo celulite e fascite necrotizante (KESIEMI, 2012).

As complicações descritas são multifatoriais e podem estar relacionadas ao desconhecimento de princípios da fisiologia respiratória, da técnica cirúrgica de implantação do dreno ou dos cuidados para manutenção do sistema de drenagem até o momento da retirada (NISHIDA, 2011). 0 uso inseguro e incorreto de tubos torácicos pode causar lesões de estruturas como vasos sanguíneos, tecido pulmonar, órgãos abdominais ou até mesmo o coração, o que pode levar a complicações fatais (PATRICK et al., 2018).

Muitos estudos mostram resultados sobre a toracostomia em pacientes vítimas de trauma, mas há poucos resultados sobre a drenagem em condições não traumáticas (MEFIRE. et al; 2014). Tendo isso em vista, o objetivo deste trabalho é descrever as principais indicações de toracostomia encontradas em condições traumáticas e não traumáticas e suas complicações.

\section{MATERIAS E MÉTODOS}

Trata-se de um estudo de série de casos, realizado no Hospital Santa Casa de Misericórdia de Barra Mansa. 0 trabalho foi aprovado pelo Comitê de Ética em Pesquisa em Seres Humanos do UniFOA, sob o CAAE 91840918.9.0000.5237.

Foi utilizada uma amostra consecutiva de pacientes submetidos à drenagem torácica, realizada no centro cirúrgico, no período de junho de 2016 a junho de 2018.

Foram incluídos no estudo, pacientes de ambos os sexos, de qualquer idade e excluídos os pacientes que realizaram drenagem pleural em outro hospital e pacientes transferidos para outro hospital no curso do tratamento.

Foram consideradas as seguintes variáveis: gênero, idade, lateralidade, indicação da drenagem, causa da lesão, tempo de drenagem e complicações.

Os dados foram tabelados e analisados, utilizando-se o programa Microsoft Excel 2017. 


\section{RESULTADOS}

0 presente estudo teve como amostra um grupo de 54 pacientes. Destes, $55.6 \%$ eram homens e $44.4 \%$ mulheres. A média de idade foi de $53,19 \pm 19.81$ anos. Quanto à lateralidade, $51.9 \%$ das drenagens foram no hemitórax esquerdo; $46.3 \%$, no direito e, $1.9 \%$, bilaterais. Em relação às indicações de drenagem, derrame pleural correspondeu a $29.6 \%$; pneumotórax a $27.8 \%$; hemopneumotórax a $20.4 \%$; hemotórax e pós-cirúrgico a 9.3\% cada e hidropneumotórax a 3.7\%. As causas das lesões foram divididas em: traumática (37.0\%); neoplásica (22.2\%); infecciosa (16.7\%); outras (14.8\%); e espontânea (9.3\%). A média do tempo de drenagem foi de $9.89 \pm 8.20$ dias. As complicações foram: pneumonia (9.3\%); empiema (5.6\%); pneumotórax residual (5.6\%); bloqueio de dreno (1.9\%); enfisema subcutâneo (1.9\%); fístula pleural (1.9\%); o restante dos pacientes $(77.8 \%)$ não tiveram complicações.

Esses dados foram apresentados na Tabela 1. 
Tabela 1 - Características dos Paciente

\begin{tabular}{|c|c|c|}
\hline Característica do Paciente & Casos & $\%$ \\
\hline Amostra & 54 & $100,0 \%$ \\
\hline \multicolumn{3}{|l|}{ Gênero } \\
\hline Masculino & 30 & $55,6 \%$ \\
\hline Feminino & 24 & $44,4 \%$ \\
\hline Média de Idade & $53,19 \pm 19.81$ & \\
\hline \multicolumn{3}{|l|}{ Lateralidade } \\
\hline Direita & 25 & $46,3 \%$ \\
\hline Esquerda & 28 & $51,9 \%$ \\
\hline Direita e Esquerda & 1 & $1,9 \%$ \\
\hline \multicolumn{3}{|l|}{ Tipo } \\
\hline Derrame pleural & 16 & $29,6 \%$ \\
\hline Pneumotórax & 15 & $27,8 \%$ \\
\hline Hemopneumotórax & 11 & $20,4 \%$ \\
\hline Pós cirúrgico & 5 & $9,3 \%$ \\
\hline Hemotórax & 5 & $9,3 \%$ \\
\hline Hidropneumotórax & 2 & $3,7 \%$ \\
\hline \multicolumn{3}{|l|}{ Causa } \\
\hline Trauma & 20 & $37,0 \%$ \\
\hline Outras & 8 & $14,8 \%$ \\
\hline Neoplasia & 12 & $22,2 \%$ \\
\hline Infecção & 9 & $16,7 \%$ \\
\hline Espontâneo & 5 & $9,3 \%$ \\
\hline Média de Tempo de Drenagem & $9,89 \pm 8,20$ & \\
\hline \multicolumn{3}{|l|}{ Complicações } \\
\hline Nenhuma & 42 & $77,8 \%$ \\
\hline Pneumonia & 5 & $9,3 \%$ \\
\hline Pneumotórax Residual & 3 & $5,6 \%$ \\
\hline Empiema Pleural & 3 & $5,6 \%$ \\
\hline Enfisema Subcutâneo & 1 & $1,9 \%$ \\
\hline Fístula Pleural & 1 & $1,9 \%$ \\
\hline Bloqueio de Dreno & 1 & $1,9 \%$ \\
\hline
\end{tabular}

Fonte: Dados da pesquisa

\section{DISCUSSÃO}

A drenagem torácica é conhecida por ser um dos procedimentos mais bem-sucedidos no tratamento de efusões pleurais (MEFIRE et al., 2014). As indicações para drenagem torácica são as mesmas para todas as instituições. O padrão de distribuição dessas indicações ou o número de drenagens associadas a uma entidade clínica específica podem, porém, variar (EDAIGBINI, 2014). 
Os resultados foram analisados, interpretados e comparados com os resultados de outros estudos como os de Edaigbini et al. (2014), Khanzada e Samad (2008) e Mefire et al. (2014).

No presente estudo, a média de idade dos pacientes foi de $53,19 \pm 19.81$. Nos estudos de Edaigbini et al. (2014), $34.85 \pm 16.72$; Khanzada e Samad (2008), $54 \pm 12.12$; Mefire et al. (2014), $34.40 \pm 7.60$. A razão entre homens e mulheres foi de 1.25, enquanto para Khanzada e Samad (2008) foi de 1.74 e para Mefire et al. (2014) foi de 0.72, o que sugere que não há associação significativa com o gênero.

A média do tempo de drenagem foi de $9.89 \pm 8.20$ dias, para Edaigbini et al. (2014), de 13.02 \pm 12.362; Khanzada e Samad (2008), de $6 \pm 2.06$; Mefire et al. (2014), de $9.3 \pm 3.8$ dias. O tempo de drenagem mais prolongado pode ser explicado pela existência de lesões associadas que demandem cuidados, como a presença de complicações que também podem aumentar o tempo de permanência do dreno (JUNIOR, 2017).

A principal indicação de toracostomia encontrada neste estudo foi o trauma (37.0\%), considerado pela Organização Mundial de Saúde como a "doença do século XXI", com grande impacto na qualidade de vida, podendo ser acidental ou intencional, causado por uma força externa, com lesões, permanentes ou não, no organismo e que, eventualmente, ameace a vida humana (PEREIRA, 2015). Nos estudos de Edaigbini et al. (2014), Khanzada e Samad (2008) e Mefire et al. (2014), as principais causas foram neoplásica $(27.5 \%)$, infecciosa $(50.5 \% ; 85.1 \%)$, respectivamente.

A taxa de complicação pós-toracostomia foi de $22.2 \%$ neste estudo, sendo a mais comum pneumonia (7.4\%), seguida de empiema e pneumotórax ( $5.6 \%$ cada). A probabilidade da ocorrência de infecção do espaço pleural é dependente de uma complexa interação entre vários fatores de risco, incluindo o grau de contaminação bacteriana, a resistência do hospedeiro, a presença de corpo estranho na cavidade, o mecanismo da lesão, entre outros (FONTANELLES, 2000). No estudo de Edaigbini et al. (2014), a taxa foi de $16.8 \%$, sendo as principais causas empiema (5.6\%) e pneumotórax (3.6\%); no de Khanzada e Samad (2008) foi de $15.2 \%$, por dobramento e enfisema (5.7\% cada); e no de Mefire et al. (2014) foi de $19.35 \%$, por deslocamento e pneumotórax. A drenagem torácica é um procedimento relativamente simples e muito usado na prática médica. 0 sucesso do procedimento depende da técnica correta durante sua realização e dos devidos cuidados pós-operatórios.

\section{CONCLUSÃO}

O trauma foi, isoladamente, a principal causa de drenagem torácica encontrada neste estudo. As drenagens torácicas por causas não traumática corresponderam a $63 \%$ dos casos, o que pode justificar a necessidade de outros trabalhos sobre toracostomia em pacientes não traumatizados.

A maior parte dos pacientes deste estudo não apresentou nenhuma complicação relacionada à drenagem torácica, sendo que a complicação mais comum foi a pneumonia, seguida de empiema pleura, enfisema, fístula e bloqueio de dreno. 


\section{REFERÊNCIAS}

ABREU, E. et al. Impacto de um Protocolo de Cuidados a Pacientes com Trauma Torácico Drenado. Disponível em: https://pdfs.semanticscholar.org/d7e0/9acb4fa74ed0514c3d624697b0a0192d1e07. pdf. Acesso em: 26 mar. 2018.

CIPRIANO, F. G.; DESSOTE, L. U. Drenagem Pleural. Disponível em: http://revista.fmrp.usp.br/2011/ vol44n1/Simp8_Drenagem\%20Pleural.pdf. Acesso em: 19 abr. 2018.

EDAIGBINI, S. A. et al. Indications and Complications of Tube Thoracostomy with Improvised Underwater Seal Bottles. Disponível em: https://www.ncbi.nlm.nih.gov/pmc/articles/PMC4141450/. Acesso em: 27 fev. 2020.

FENILI, R.; ALCACER, J. A. M.; CARDONA, M. C. Traumatismo Torácico - Uma Breve Revisão. Disponível em: http://www.acm.org.br/revista/pdf/artigos/31.pdf. Acesso em 19 abr. 2018.

FONTELLES, M. J. P.; MONTOVANI, M. Trauma Torácico: Fatores de Risco de Complicações Pleuropulmonares Pós-Drenagem Pleural Fechada. Disponível em: http://www.scielo.br/pdf/rcbc/v27n6/08.pdf. Acesso em: 19 abr. 2018.

GENNARI, T. D.; KOIZUMI, M. S. Determinação do Nível de Gravidade do Trauma. Disponível em: http:// www.scielo.br/scielo.php?pid=S0034-89101995000500001\&script=sci_arttext. Acesso em: 19 abr. 2018.

JUNIOR, C. A. B. et al. Perfil dos Pacientes Vítimas de Trauma Torácico Submetidos à Drenagem de Tórax. Disponível em: http://www.scielo.br/pdf/rcbc/v44n1/pt_0100-6991-rcbc-44-01-00027.pdf. Acesso em: 19 abr. 2018.

KHANZADA, T. W., SAMAD A. Indications and complications of tube thoracostomy performed by general surgeons. Disponível em: https://pubmed.ncbi.nlm.nih.gov/18297976/. Acesso em 27 fev. 2020.

KESIEMI, E. B. et al. Tube Thoracostomy: Complications and Its Management. Disponível em: https:// www.hindawi.com/journals/pm/2012/256878/. Acesso em: 19 abr. 2018.

KWIATT, M. et al. Thoracostomy Tubes: A Comprehensive Review of Complications and Related Topics. Disponível em: https://www.ncbi.nlm.nih.gov/pmc/articles/PMC4093965/. Acesso em: 19 fev. 2018.

MEFIRE, A. C.; FOKOU, M.; DIKA, L. D. Indications and morbidity of tube thoracostomy performed for traumatic and non-traumatic free pleural effusions in a low-income setting. Disponível em: https://www. ncbi.nlm.nih.gov/pmc/articles/PMC4258205/\#CIT0010. Acesso em: 27 fev. 2020.

MENDES, C. A.; HIRANO, E. S. Fatores Preditores de Complicações da Drenagem de Tórax em Pacientes Vítimas de Trauma. Disponível em: http://www.scielo.br/scielo.php?script=sci_arttext\&pid=S010069912018000200154\&lng=pt\&nrm=iso\&tlng=pt. Acesso em: 23 abr. 2020.

NISHIDA, G. et al. Cuidados com o sistema de drenagem torácica em adultos internados no Hospital Universitário Regional de Maringá, Estado do Paraná, Brasil. Disponível em: http://periodicos.uem.br/ ojs/index.php/ActaSciHealthSci/article/viewFile/8877/pdf. Acesso em: 19 abr. 2018. 
PATRICK, H; JULIAN, O. et al. Development and validation of an objective assessment scale for chest tube insertion under 'direct' and 'indirect' rating. Disponível em: https://www.ncbi.nlm.nih.gov/pmc/ articles/PMC6307220/\#!po=28.3333. Acesso em: 27 fev. 2020.

PEREIRA, R. Traumatismos Torácicos - Revisão. Disponível em: https://estudogeral.sib.uc.pt/jspui/ bitstream/10316/30716/1/Rute\%20Pereira\%20-\%20Traumatismo\%20tor\%C3\%A1cico\%20-\%20 vers\%C3\%A30\%20final.pdf. Acesso em: 26 mar. 2018.

VILKKI, V. A.; GUNN, J.M. Complications related to tube thoracostomy in Southwest Finland hospital district between 2004 and 2014. Disponível em: https://journals.sagepub.com/doi/full/10.1177/14574 96919857262?url_ver=Z39.88-2003\&rfr_id=ori\%3Arid\%3Acrossref.org\&rfr_dat=cr_pub\%3Dpubmed. Acesso em: 27 fev. 2020. 